\title{
Android Based Smart Door Lock Mechanism for Managing Security of Disabled People
}

\author{
Anuradha Krishna1, Dr. Sweta ${ }^{2}$ \\ ${ }^{1}$ Lecturer (Senior Scale), New Government Polytechnic, Patna1 \\ 2Assistant Professor, Birla Institute of Technology, Mesra, Ranchi, Patna Campus, Patna2 \\ anuradha.krishna4@gmail.com, sweta.tiwary@bitmesra.ac.in
}

\begin{abstract}
Security has always been of utmost importance everywhere. People want their houses, stores, warehouses etc. to be secure at all times. To make this possible and much easier, smart door locking mechanism was brought into the picture. A smart door lock can help us to ensure that our houses are locked and we can check this from anywhere with only a mobile phone in our hands. This is the perfect example of managing home security without the requirement of being there. This can be implemented by using a Bluetooth Hc- 05 module to connect an Arduino UNO board or a microcontroller to an Android app or by using IOT depending on the range of access required by the users. This smart home automation system can specifically manage life a little easier for people having some form of physical disability.
\end{abstract}

Keywords

Article Received: 10 August 2020, Revised: 25 October 2020, Accepted: 18 November 2020

\section{Introduction}

Smart Home Technology refers to all those technologies that can improve by managing our way of living and make life easier for everyone. In today's world where every other person is equipped with a smart phone, the implementation of these technologies has become easier and are also easily available to the masses. One such home automation system is the smart door locking mechanism.

Initially the idea of using a door lock originated way back with the sole purpose of security. Over the years, locks have become more and more complex starting off the basic padlock. The padlock did not have to be attached to the doors but could be used whenever necessary. With advancement in technology, deadbolts or knob locks came into the picture which are permanently attached to the doors. Technology become demand of the day since in the current world we are moving more and more towards smart technology, the smart door locking mechanism also came into the picture.

The smart lock can be implemented by using either Bluetooth or IOT technology. Of course, if we bring the idea of internet to operate something, the costs surge comparatively. On the other hand, using Bluetooth reduces the costs to a great extent. Since the locking or unlocking of doors would be restricted to only a few metres within the radius of the house, we preferred using this.

The topic uses this Bluetooth technology to maintain the connection between our hardware and software. The hardware used is an Arduino Board and software is an Android version on a smart phone to run the app that controls the locking and unlocking. So for managing security one need to select the most appropriate one by cost and benefit analysis.

\section{Android App}

The Android app for the smart door lock is designed using React Native framework, an open mobile application framework developed by Facebook.

\section{Arduino:}

Arduino board is a microcontroller that takes an input as a code and converts into a hardware-based output like turning on LED or rotating a motor etc.

\section{Purpose:}

The smart door locking mechanism has been developed to ensure security of houses, stores etc. and also managing life a little easier for the people suffering from some sort of disability. This technology is simple to use for the common masses and also comparatively low cost. The design proposed in this project uses Bluetooth technology to establish connection between the app and the door. Bluetooth technology is one of the cheapest available technologies and is very useful in applications that require a short-range communication of up to 100 metres.

The locking system is something that is a necessity for all the people and should be designed such that it can be accessed by the user both from the inside and outside. The user can install the app on their phone and send a signal to the door via Bluetooth to lock or unlock it. The entire access lies in the hands of the user and hence covers the security problem.

\section{Summary:}

The topic comprises of an Arduino board, a Bluetooth module and an Android phone. On receiving the correct password from the user, the app gives us a list of all the Bluetooth devices in the vicinity. We connect it to our 
hardware and then it directs us to choose whether we want to lock or unlock it. Depending on the choice, it sends a signal to the Arduino which further sends a signal to the motor to rotate and perform the desired action.

The flow chart given below represents the work flow of the entire app and the connection it maintains with Arduino to do the work.

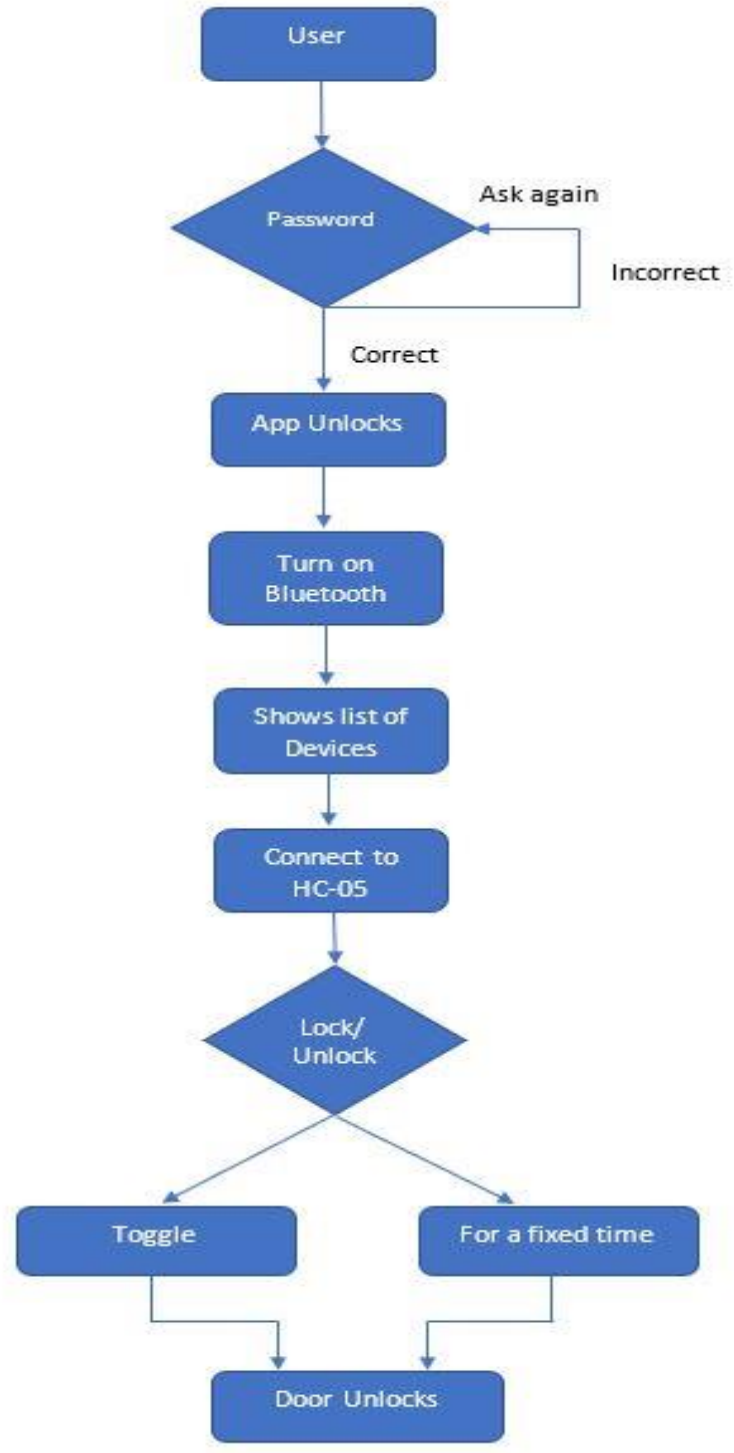

Fig: Flow Chart

\section{Motivation}

The basic purpose of this paper is to serve the people who are handicapped and are unable to use their hands or legs. Hence this is the motivation behind the project and the features added in the app fulfil them. We have added voice commands for all actions to benefit people who cannot see or use their hands and the basic concept of locking and unlocking doors from a particular place is to benefit those who are not able to walk. They do not have their dependency on others by the use of this app.

The second part of the motivation is obviously the security purpose. In today's world security is a major concern and we aim to take a step towards solving it. The locking can be controlled only from the app and to log in to the app you need to know the password which makes sure that the access lies only in the hands of the user. This is the way we can manage security.

\section{Literature Survey}

Before the advent of technology, the locks used in households and elsewhere for safety purposes were mechanical in nature and consisted of a unique key that twisted the levers and gears inside it to open it. These were designed by blacksmiths mostly.

But with the advancement in technology over time, more robust locks came into picture to fulfil the increasing needs of security and privacy. These locks used the concept of conventional locks and integrated them with digital technologies. In this digital lock a particular digital key in the form of password is to be used. Various technologies like Bluetooth, IOT, etc. are basically used in this field depending on the availability of internet, cost and distance requirements.

Chi-Huang Hung et al [1] proposed a design which ingrates the close by transmission reader of any Android device with the door lock command structure to supply an easy-to-use performing system. Their framework gave a 3-way control mode to match passwords and amplify security thereby. They also added new characteristics like sleep and standby for saving power to increase duration of operation of the system.

Henry Clinton et al [2] proposed a novel approach which deals with security-based system to provide a smart door lock system. In his work, he uses 4 modules which are implemented on an electronic door lock with a gear motor within it which facilitates the mechanical system of lock and unlock. To control the overall system, an Arduino MEGA microcontroller has been deployed. The 4 modules are fingerprint sensor, messaging module, passcode module using Keypad and Voice Controller module which involves Bluetooth module.

N. H. Ismail et al [3] proposed a system that is meant to help differently-abled people in their houses. In this paper, Bluetooth technology is used to establish connection between the Android phone and microcontroller. Relay board is connected to the circuit and it is connected to Arduino Controller board. By connecting all this, it can be administered by available Bluetooth to provide remote access from smartphone. He also addressed the buildout and the purpose of the App on how to aid disabled people.

Dennis Sullivan et al [4] developed a paper which allows users to control numerous gadgets remotely from a smartphone. It entails the use of Bluetooth connection and a microcontroller such as Arduino UNO Rev 3. The intention behind this paper is to develop an Android app to control home appliances using AC power.

Adnan Ibrahim et al [5] introduced the design of a GSM based computerized door lock safety system using a PIC platform. In this, a password was used to open or close the door by deploying a motor. A warning message is sent if more than 3 unsuccessful attempts are made to enter the correct password. To demonstrate the proposed system, a 3D gauged replica of a house with doors administered by a motor is fabricated. 


\section{Contribution (Proposed Work)}

Our approach to design a smart lock is very simple as Bluetooth connection is used. It comprises of both hardware and software requirements which are easily available and is also cost effective. The hardware requirements are an Arduino uno board, a HC-05 Bluetooth module, a motor like SG-90 and a few jumper wires. The software requirements comprise of an Arduino IDE to run the Arduino code and an android phone on which the app can run.

\section{Hardware:}

The hardware circuit connections are established by using the Arduino board, Bluetooth module and servo motor.

\section{Software:}

The android app is built using React Native, an open-source mobile application framework created by Facebook. The design and user interface of the app is kept simple and easy to use. The app uses Bluetooth serial to connect it with the available Bluetooth devices in the range of our android device. Also, it has an inbuilt app-lock feature including both pattern lock and voice password which makes it more secure. It also uses voice commands for all functions to take inputs making it further more user friendly.

\section{Working:}

After making the required connections we run the code on the IDE that results in the lock-unlock procedure. The entire process can be divided into three parts:

\section{Password Authentication:}

When the user opens the app, he/she is visited by a password authentication which ensures that unauthorised access is blocked. When the user enters the right password, only then the app gives them access to the Bluetooth enabling feature. Whereas if wrong password is entered, an error message is popped-up, requesting the user to re-enter the password.

We have also added a voice feature to enter the password instead of typing it.

\section{Bluetooth Connection:}

When the user enters the correct password then he is directed to the feature where toggling the Bluetooth from within the app is given. On switching ON Bluetooth, we get a list of available devices which are in the range of our android device. From this list we can choose the Arduino and connection is established using the $\mathrm{HC}-05$ module.

\section{Lock-Unlock:}

Having established the connection, the user can select whether they want to lock the door or unlock it. This sends a signal to the servo motor which changes its angle from zero to ninety degrees and vice versa depending on the command performing the required action. There are two options that can be used:

- Toggle ON/OFF: This implies that the user can mechanically switch between on and off by pressing this button.

- For a fixed duration: By using this option the unlocked door will lock itself after the specified period of time even if the user forgets to do so. This feature further enhances the security constraint.

\section{Implementation}

For this both software and hardware requirements needed:

\section{Software Requirements:}

- $\quad$ Arduino IDE

- $\quad$ Android Version 8 and above

\section{Hardware Requirements:}

- $\quad$ Arduino UNO Board

- $\quad$ Bluetooth Module (HC-05/HC-10)

- $\quad$ SG-90 Motor

- Jumper wires

- $\quad$ Breadboard

When the app opens the icon splashes forward and the diminishes to move onto the page requesting for password.

\section{Password Authentication:}

On opening the app password authentication takes place. We can select a pattern for the password or enter a password using voice commands. Only the correct password enables the user to $\log$ in to the system.

On the home page we have two options of either locking through toggling or to open the door for a fixed duration.

\section{Bluetooth connection:}

On selecting either of the options, we are led to a page that enables us to connect any device in the vicinity of our mobile phone using Bluetooth. On switching it on a list of devices are enlisted and we can select our Bluetooth module from amongst the list to establish connection with the hardware.

\section{Lock-Unlock:}

There are two ways to open and close the door. When the connection is established the user can send either a voice command to open the door for a fixed duration of time or can toggle between lock and unlock. The voice command feature enables it to be used by people who are handicapped in their hands. Also, it adds more security by locking the door itself in case the user forgets to do so.

\section{Results}

Various wireless protocols are available for smart home automation systems. Some of the most used ones are- Wi-Fi, 
Bluetooth and Z-wave or Zigbee. We have used Bluetooth low energy or BLE for our smart lock mechanism.

Using BLE has various advantages in our project because we need to control the lock from a short range only and hence Bluetooth is more suited than Wi-Fi. Also, the cost of using BLE over Wi-Fi is beneficial and hence our project can be used by all irrespective of classes. The security aspect of BLE is also highest among the three. Next, the power consumption of BLE is also very less in comparison. Losing only on the range aspect we have security, cost and low power consumption benefits of BLE. Hence it is most effective for designing a smart door lock for a household. A table comparing the different features of the three protocols as well as bar graphs are drawn below:

\begin{tabular}{|c|c|c|c|}
\hline Type & Wi-Fi & Bluetooth & ZigBee \\
\hline $\begin{array}{l}\text { Operating } \\
\text { Frequency }\end{array}$ & $\begin{array}{l}2.4 \mathrm{GHz} \\
5 \mathrm{GHz}\end{array}$ & $2.4 \mathrm{GHz}$ & $\begin{array}{l}868 \mathrm{MHz}, \\
915 \mathrm{MHz}, \\
2.4 \mathrm{GHz}\end{array}$ \\
\hline $\begin{array}{l}\text { Transmission } \\
\text { rate }\end{array}$ & $\begin{array}{l}11 \text { Mbps, } \\
54 \text { Mbps }\end{array}$ & $720 \mathrm{Kbps}$ & $\begin{array}{l}20 \mathrm{Kbps}, \\
40 \mathrm{Kbps}, \\
250 \mathrm{Kbps}\end{array}$ \\
\hline Network node & 32 & 7 & $65 \mathrm{~K}$ \\
\hline Power & $10-50 \mathrm{~mA}$ & $20 \mathrm{~mA}$ & $5 \mathrm{~mA}$ \\
\hline Security & Low & High & Middle \\
\hline Cost & $25 \$$ & $2-5 \$$ & $5 \$$ \\
\hline $\begin{array}{l}\text { Main } \\
\text { applications }\end{array}$ & $\begin{array}{l}\text { Date } \\
\text { Transmission }\end{array}$ & $\begin{array}{l}\text { Date and } \\
\text { Voice } \\
\text { Transmission }\end{array}$ & $\begin{array}{l}\text { Monitoring, } \\
\text { Control }\end{array}$ \\
\hline
\end{tabular}

\section{Comparison of different Wireless Protocols}
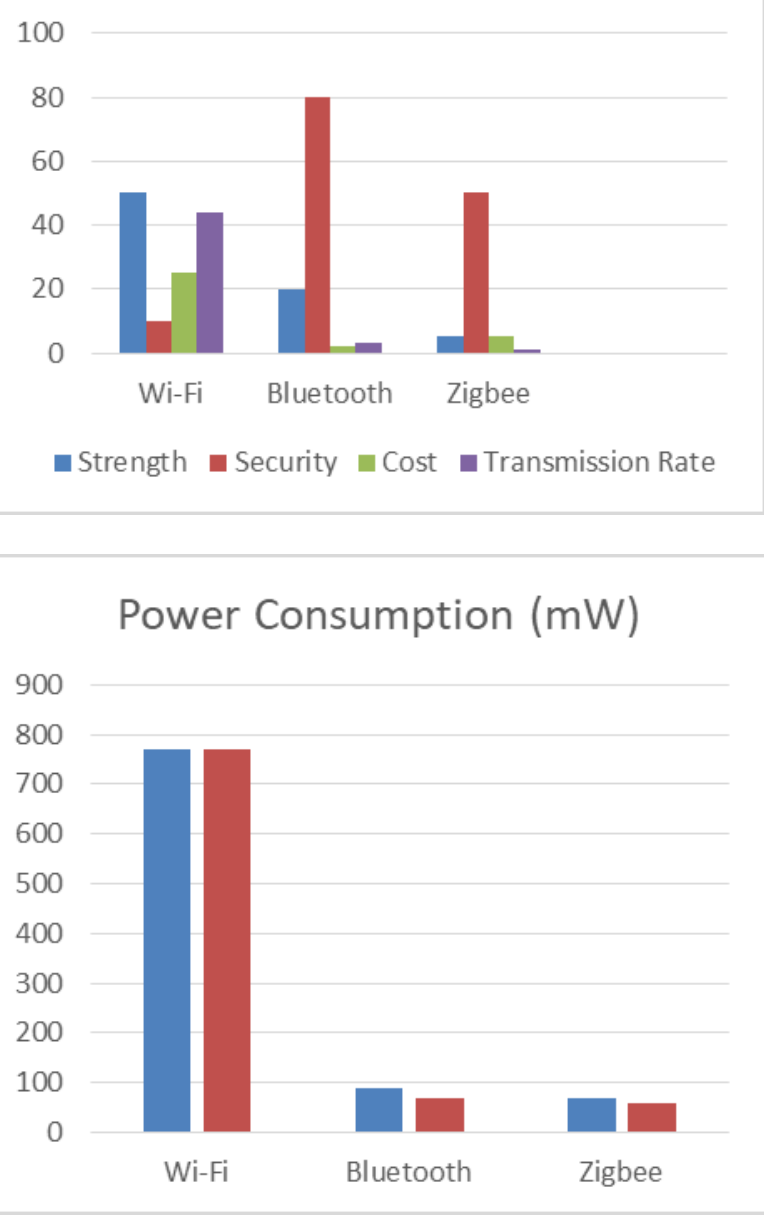

\section{Conclusion}

In this paper aims in designing a system that increases the comfort level of the users by enabling them to lock and unlock the doors without having to get up from their positions, increases security of households and specially help the people with various forms of disability. This would prove the smart way of managing security. There is no longer any direct contact between the door and the user. This paper fulfils its three main criteria - privacy, security and comfort.

\section{References}

[1] Hung, C. H., Bai, Y. W., \& Ren, J. H. (2015, September). Design and implementation of a single button operation for a door lock control system based on a near field communication of a smartphone. In 2015 IEEE 5th International Conference on Consumer Electronics-Berlin (ICCE-Berlin) (pp. 260261). IEEE.

[2] Hung, C. H., Bai, Y. W., \& Ren, J. H. (2015, June). Design and implementation of a door lock control based on a near field communication of a smartphone. In 2015 IEEE International Conference on Consumer Electronics-Taiwan (pp. 45-46). IEEE.

[3] Henry Clinton, S., Rakshantha, A., Koperundevi, G., Suriyakumar, P., \& Vetrichelvi, G. C-Sey-Smart Door Lock System.

[4] Ismail, N. H., Tukiran, Z., Shamsuddin, N. N., \& Saadon, E. I. S. (2014, November). Android-based home door locks application via Bluetooth for disabled people. In 2014 IEEE International Conference on Control System, Computing and Engineering (ICCSCE 2014) (pp. 227-231). IEEE.

[5] Sullivan, D., Chen, W., \& Pandya, A. (2017, June). Design of remote control of home appliances via Bluetooth and Android smart phones. In 2017 IEEE International Conference on Consumer Electronics-Taiwan (ICCE-TW) (pp. 371372). IEEE. 
[6] Ibrahim, A., Paravath, A., Aswin, P. K., Iqbal, S. M., \& Abdulla, S. U. (2015, December). GSM based digital door lock security system. In 2015 International Conference on Power, Instrumentation, Control and Computing (PICC) (pp. 1-6). IEEE.

[7] Kavde, S., Kavde, R., Bodare, S., \& Bhagat, G. (2017, February). Smart digital door lock system using Bluetooth technology. In 2017 International Conference on Information Communication and Embedded Systems (ICICES) (pp. 1-4). IEEE.

[8] Hadis, M. S., Palantei, E., Ilham, A. A., \& Hendra, A. (2018, March). Design of smart lock system for doors with special features using bluetooth technology. In 2018 International Conference on Information and Communications Technology (ICOIACT) (pp. 396-400). IEEE.

[9] Doh, O., \& Ha, I. (2015). A digital door lock system for the internet of things with improved security and usability. Advanced Science and Technology Letters, 109(Security, Reliability and Safety 2015), 33-38.

[10] Tiwari, S., Thakur, S., Shetty, D., \& Pandey, A. (2018, April). Smart security: remotely controllable doorlock. In 2018 Second International Conference on Inventive Communication and Computational Technologies (ICICCT) (pp. 979-984). IEEE.

[11] Park, Y. T., Sthapit, P., \& Pyun, J. Y. (2009, January). Smart digital door lock for the home automation. In TENCON 2009-2009 IEEE Region 10 Conference (pp. 1-6). IEEE.

[12] De Camargo Silva, L., Samaniego, M., \& Deters, R. (2019, October). IoT and Blockchain for Smart Locks. In 2019 IEEE 10th Annual Information Technology, Electronics and Mobile Communication Conference (IEMCON) (pp. 0262-0269). IEEE.

[13] Nadiya, U., Rizqyawan, M. I., \& Mahnedra, O. (2019, November).
Blockchain-based Secure Data Storage for Door Lock System. In 2019 4th International Conference on Information Technology, Information Systems and Electrical Engineering (ICITISEE) (pp. 140-144). IEEE.

[14] Dabhade, J., Javare, A., Ghayal, T., Shelar, A., \& Gupta, A. (2017). Smart Door Lock System: Improving Home Security using Bluetooth Technology. International Journal of Computer Applications, 160(8).

[15] Kamelia, L., Noorhassan, A., Sanjaya, M., \& Mulyana, W. E. (2014). Doorautomation system using bluetooth-based android for mobile phone. ARPN Journal of Engineering and Applied Sciences, 9(10), 1759-1762. 